\title{
Expression of melanocortin receptors in human prostate cancer cell lines: MC2R activation by ACTH increases prostate cancer cell proliferation
}

\author{
SALY HAFIZ ${ }^{1,2}$, JOHN C. DENNIS ${ }^{1}$, DEAN SCHWARTZ ${ }^{1}$, ROBERT JUDD ${ }^{1}$, \\ YA-XIONG TAO $^{1}$, KAMEL KHAZAL ${ }^{2}$, BENSON AKINGBEMI ${ }^{1}$, XIU-LEI MO ${ }^{1}$, \\ ASIM B. ABDEL-MAGEED ${ }^{3}$, EDWARD MORRISON ${ }^{1}$ and MAHMOUD MANSOUR ${ }^{1}$ \\ ${ }^{1}$ Department of Anatomy, Physiology, and Pharmacology, College of Veterinary Medicine, \\ Auburn University, Auburn, AL; ${ }^{2}$ Department of Biomedical Sciences, College of Veterinary \\ Medicine, Tuskegee University, Tuskegee, AL; ${ }^{3}$ Urology and Oncology Research Laboratories, \\ Tulane University Health Sciences Center, Tulane Cancer Center, New Orleans, LA, USA
}

Received April 3, 2012; Accepted May 8, 2012

DOI: $10.3892 /$ ijo.2012.1574

\begin{abstract}
The melanocortin receptors (MCRs 1-5) are $\mathrm{G}$ protein coupled-receptors (GPCRs) that regulate food intake, inflammation, skin pigmentation, sexual function and steroidogenesis. Their peptide ligands, the melanocortins, are $\alpha-, \beta$ - and $\gamma$-melanocyte-stimulating hormone and adrenocorticotropic hormone (ACTH) all of which are secreted from the anterior pituitary gland under hypothalamic control. MC2R binds ACTH but has no affinity for the other melanocortins and is, thereby, pharmacologically different from MCRs that bind those ligands. Evidence suggests that elevated GPCRs transactivate the androgen receptor (AR), the critical mediator of prostate cell growth, and consequently promote prostate cancer cell proliferation. It may be that reduced central melanocortin signaling is coincidental with reversal of prostate cancer cachexia, but no data are available on the expression of, or the role for, MCRs in prostate cancer. Here, we show that MCR (1-5) mRNAs are expressed in androgen-dependent LNCaP and androgen-independent PC3 and DU-145 human prostate cancer cell lines. Further, MC2R, the specific target of ACTH, is expressed in LNCaP, PC3 and DU-145 cells. Among the several synthetic MCR peptide ligands that we
\end{abstract}

Correspondence to: Dr Mahmoud Mansour, Department of Anatomy, Physiology, and Pharmacology, College of Veterinary Medicine, Auburn University, Auburn, AL, USA

E-mail: mansoma@auburn.edu

Abbreviations: MCRs, melanocortin receptors; ACTH, adrenocorticotropic hormone; AR, androgen receptor; MC2R, melanocortin receptor 2; GPCRs, G protein coupled-receptors; PCa, prostate cancer

Key words: melanocortin receptors, adrenocorticotropic hormone, androgen receptor, melanocortin receptor 2, G protein coupledreceptors, prostate cancer used, only ACTH promoted concentration-dependent cell proliferation in the three cell lines as shown by MTT cell proliferation assay. In LNCaP cells, the effect was additive with testosterone stimulation and was partially blunted with SHU9119, a non-selective MCR antagonist. In the same cells, ACTH induced cAMP production and increased AR nuclear labeling in immunocytochemical assays. Our observations suggest that MC2R is involved in prostate carcinogenesis and that targeting MC2R signaling may provide a novel avenue in prostate carcinoma treatment.

\section{Introduction}

Prostate cancer (PCa) is a leading cause of cancer mortality among men in the United States and is the second leading cause of cancer mortality in men worldwide (1). Current treatments include hormonal therapy, targeted radiation, and radical prostatectomy. The only alternative is watchful waiting. The limitations and side-effects associated with these therapies, however, warrant the development of new therapies (2).

Testosterone $(\mathrm{T})$ regulates normal cell growth in the prostate gland and is secreted by testicular Leydig cells. The action of $\mathrm{T}$ is mediated by the androgen receptor (AR), a nuclear receptor expressed in the stromal and luminal prostate gland epithelial cells. Androgen-dependent and independent neoplastic PCa cells depend on T, its more potent $5 \alpha$-reductase metabolite, $5 \alpha$-dihydrotestosterone (DHT), and AR (3). Huggins (4) demonstrated PCa dependency on T and, based on his early findings that showed reduced prostate growth following removal of androgen, local and metastatic cancers are currently treated with endocrine therapies aimed at reducing androgen production and/or blocking AR with anti-androgens. These therapies limit tumor growth and reduce tumor mass but the cancer may recur as androgeninsensitive (AI) disease (5).

Multiple direct and indirect mechanisms are thought to cause the AI state through changes in AR activity (6-8). These 
changes include AR signaling modulation by a kinase receptor such as human epidermal growth factor receptor 2 (HER-2/ neu) tyrosine kinase (9); overexpression of, or mutations in, the AR gene (10); increased AR sensitivity (11); or activation of the AR by growth factors such as insulin-like growth factor 1 , epidermal growth factor and kerationcyte growth factor $(6,12)$. Additional indirect and equally important mechanisms by which PCa cell growth circumvent androgen-dependency involve overexpression of GPCRs $(13,14)$. An effective cure for AI is currently unavailable despite the intense research in this area (15).

Since mutations in the AR are responsible for a small number of prostate cancers, indirect mechanisms are likely to be involved in the majority of androgen-refractory cases. One such mechanism is activation and overexpression of GPCRs (8). Several studies showed that GPCRs are overexpressed in various cancer types and contribute to tumor growth when activated by circulating or locally produced peptide ligands (13). These ligands induce cell proliferation and prevent apoptosis of hormone-independent PCa cells, which suggest a critical role in PCa progression (14,16-20). Normal GPCR functions are altered in malignant cells leading to autonomous cell proliferation (13). Hence, GPCR-targeted therapy for the treatment of $\mathrm{PCa}$ is actively pursued (21).

GPCR actions in PCa involve cell growth enhancement via ERK6, a mitogen-activated protein kinase-related serine/ threonine kinase (MAPK)(22). ERK can phosphorylate AR at several sites thereby upregulating AR target genes (13). GPCRs that signal through stimulatory $G$ proteins $\alpha(G s \alpha)$ can also transactivate AR through cyclic adenosine monophosphate (cAMP) production $(23,24)$ and protein kinase A (PKA)(25). In addition, the activated Gs $\alpha$ subunit can directly activate AR and act synergistically with low androgen concentrations to increase AR activity in PCa cells (24).

Several studies suggest a role for at least some of the MCRs in cancer development. An association between MC1R and PCa risk was reported in human (26) and central MCR blockade with SHU9119-reversed PCa anorexia in Wistar rats (27). Additionally, MC5R is widely expressed in normal rodent exocrine glands including the prostate (28).

$\mathrm{MC} 2 \mathrm{R}$, activated by $\mathrm{ACTH}$, is involved in androgen and steriodogenic enzyme synthesis. It is normally expressed in the adrenal gland cortex and epidermis as well as in basal cell carcinoma tissue and cultured melanocytes. As with other GPCRs, MC2R binding ACTH activates adenylyl cyclase with subsequent production of cAMP. Cyclic AMP can then directly activate or interact with various proteins such as ionchannels, transcription factors, guanine nucleotide exchange factors, and protein kinases such as PKA. PKA and cAMP play critical roles in the progression and differentiation of prostate carcinogenesis (25). Activation of MC2R can also result in phosphorylation of MAPK and the MC2R accessory protein isoform $\beta$ (MRAP- $\beta$ ) in cells stably expressing MC2R (29). Similarly, ectopic production of ACTH, the putative ligand for $\mathrm{MC} 2 \mathrm{R}$, has been reported in cases of prostate carcinoma associated with Cushing's syndrome (30).

Currently, the role of MCRs in PCa is unclear. The objective of this study was to investigate the MCRs expression in human PCa cell lines and their effect on cell proliferation, with particular emphasis on MC2R.

\section{Materials and methods}

Reagents. ACTH and DHT were obtained from Sigma (St. Louis, MO). Melanocortin peptides, NDP-MSH (a potent $\alpha$-MCR analogue for all MCRs except MC2R) and THIQ (highly selective MC4R agonist), D-Trp ${ }^{8}-\gamma$-MSH (highly selective MC3R agonist), and SHU9119 (a universal MCR blocker) were obtained from Tocris Bioscience, Ellisville, MO. A selective MC4R inverse agonist (Ipsen 5i) was synthesized by Enzo Life Sciences International Inc., Plymouth Meeting, PA. RPMI-1640 media with and without phenol red, Dulbecco's phosphate buffered saline (DPBS), without calcium and magnesium, Forskolin (cAMP inducer), and penicillin streptomycin antibiotics were purchased from Lonza (Portsmouth, $\mathrm{NH})$. Heat-inactivated and charcoal-dextran-treated fetal calf serum (FCS) was obtained from Atlanta Biological (Atlanta, GA). Cell culture flasks and other disposable cell culture supplies were purchased from VWR International, LCC (Atlanta, GA). MTT cell viability assay kits were purchased from the American Type Culture Collection (ATCC). Primary antibodies and control peptides for MC2R (SA-639), and AR (Sc-816) were obtained from Enzo Life Science (Farmingdale, NY) and Santa Cruz Biotechnology (Santa Cruz, CA), respectively. Dylite 488 conjugated goat anti-rabbit IgG (Jackson Immunoresearch Laboratories, PA) was used for the detection of primary binding in immunocytochemistry assays.

Cell culture. The human prostate normal epithelial cells, RWPE-1, and human prostate adenocarcinoma cell lines, LNCaP, PC3, and DU-145, were obtained from the American Type Culture Collection (Manassas, VA). The RWPE-1 cells were propagated in base medium containing bovine pituitary extract and human recombinant epidermal growth factor (Invitrogen, Carlsbad, CA). PCa cell lines were maintained in RPMI-1640 media supplemented with $1 \%(\mathrm{v} / \mathrm{v})$ streptomycin-penicillin antibiotics (Invitrogen), and 10\% (v/v) heat-inactivated FCS. Cells were grown in T-75 vented filter cap tissue culture flasks until they reached approximately $75-90 \%$ confluency at $37^{\circ} \mathrm{C}$ in a humidified incubator under a $5 \% \mathrm{CO}_{2}$ atmosphere. Detachment of the cells from the T-75 flasks was accomplished with trypsin after the cells were washed with DPBS to remove residual serum. Cells were then counted using an automated coulter cell counter and cultured at the desired concentration. For culture cell proliferation/ viability experiments, ligands were diluted in PBS-BSA and added in $20 \mu 1$ volumes containing various concentrations. Twenty-four hours before treatments, cells were depleted of androgen by replacing growth media with phenol red-free RPMI-1640 supplemented with 5\% charcoal-dextran-treated FCS. Treatment peptides were replenished every $24 \mathrm{~h}$ for $72 \mathrm{~h}$. Cells were seeded at a density of $2 \times 10^{5}$ cells per well when grown in 6-well culture plates (for protein and RNA isolation), and at $2 \times 10^{3}$ cells per well when grown in flat-bottom 96-well plates for MTT cell viability assay. Passage number was recorded with each split and cells were used between passages 5 and 38.

MTT cell viability assay. Cells in log-phase growth were harvested via trypsinization and counted using a coulter counter. The effect of test drugs on cell viability/ 
Table I. Expected PCR product size and accession nos. of human MCR genes.

\begin{tabular}{lccc}
\hline Gene symbol & Expected PCR product size & RefSeq accession no. & Reference positions \\
\hline MC1R & 179 & NM_002386.3 & $2933-2951$ \\
MC2R & 159 & NM_000529.2 & $180-202$ \\
MC3R & 146 & NM_019888.3 & $639-658$ \\
MC4R & 186 & NM_005912.2 & $717-741$ \\
MC5R & 90 & NM_005913.1 & $103-121$ \\
\hline
\end{tabular}

proliferation was determined using the 3-(4,5-dimethylthiazol2-yl)-2-5-diphenyltetrazolium bromide (MTT) cell viability assay ATCC kit according to the manufacturer's instructions. This colometric assay measures the reduction of a tetrazolium component (MTT) into an insoluble formazan product by the mitochondria of metabolically active cells. Forty-eight to $72 \mathrm{~h}$ after test drugs were added (eight replicates per treatment), $10 \mu \mathrm{l}$ MTT reagent was added per well for $2 \mathrm{~h}$ at $37^{\circ} \mathrm{C}$. Next, $100 \mu \mathrm{l}$ detergent solution was added to lyse the cells and solubilize colored crystals. Plates were then incubated in the dark for $6 \mathrm{~h}$ at room temperature (RT). Optical density (OD) was obtained using Spectramax Plus microtiter plate reader at $570 \mathrm{~nm}$ wavelength. The amount of purple color produced in this test is directly proportional to the number of viable and metabolically active cells. The effect on cell proliferation/viability was calculated based on three independent experiments.

Conventional and real-time PCR. Total RNA was isolated using TRIzol reagent (Invitrogen-Life Technologies Inc., Carlsbad, CA, USA), according to the manufacturer's protocol and as described in our previous study (31). RNA concentrations were determined at $260 \mathrm{~nm}$ wavelength and the ratio of 260/280 was obtained using UV spectrophotometry (DU640, Beckman Coulter, Fullerton, CA, USA). Samples with 260/280 ratio of $\geq 1.8$ were used. First strand synthesis was performed with SABiosciences first strand kit C-03 (SABiosciences, Frederic, MD, USA). Conventional PCR was used initially to determine the presence of MCR (1-5) genes in LNCaP, PC-3, and DU-145 using validated human primer sets obtained from SABiosciences (Table I). RT-PCR was performed using ReactionReady ${ }^{\mathrm{TM}}$ HotStart 'Sweet' PCR master mix (SABiosciences). PCR products were analyzed in parallel with GAPDH housekeeping gene. Final end PCR products were fractionated and visualized on $2 \%$ ethedium bromide-stained agarose gel. Next, real-time PCR was used to determine the expression level of the MCRs (1-5) in LNCaP, PC-3 and DU-145 cancer cells relative to RWPE 1 normal prostate epithelial cells. The assay was carried out as we previously described (31). Briefly, reactions were performed in $25 \mu \mathrm{l}$ reaction mixture containing $12.5 \mu \mathrm{l}$ RT2 real-time SYBR/ Fluorescein Green PCR master mix with final concentrations of $10 \mathrm{mM}$ Tris- $\mathrm{HCl}, 50 \mathrm{mM} \mathrm{KCl}, 2.0 \mathrm{mM} \mathrm{MgCl}, 0.2 \mathrm{mM}$ dNTPs, and 2.5 units of HotStart Taq DNA polymerase (SABiosciences), $1 \mu \mathrm{l}$ first strand cDNA, $1 \mu \mathrm{l}$ RT2 validated primer sets, and 10.5 $\mu \mathrm{l}$ PCR-grade water. Reactions were run in 96-well PCR plates using Bio-Rad PCR cycler (Bio-Rad, MyiQ, Hercules, CA, USA). Reactions were run in duplicates and the results were normalized to GAPDH. The amplification protocol was set at $95^{\circ} \mathrm{C}$ for $15 \mathrm{~min}$, and 40 cycles each at $95^{\circ} \mathrm{C}$ for $30 \mathrm{sec}, 55^{\circ} \mathrm{C}$ for $30 \mathrm{sec}$, and $72^{\circ} \mathrm{C}$ for $30 \mathrm{sec}$.

Western blotting (WB). WB was performed as previously described by us (31). Briefly, cell lysates were prepared by homogenization in RIPA buffer $(150 \mathrm{mM}$ sodium chloride, $50 \mathrm{mM}$ Tris- $\mathrm{HCl}, \mathrm{pH} 7.4,1 \mathrm{mM}$ ethylenediaminetetraacetic acid, $1 \mathrm{mM}$ phenylmethylsulfonyl flouride, $1 \%$ Triton X-100, $1 \%$ sodium deoxycholic acid, $0.1 \%$ sodium dodecylsulfate) containing $5 \mu \mathrm{g} / \mathrm{ml}$ of aprotinin, and $5 \mu \mathrm{g} / \mathrm{ml}$ of leupeptin. Cell debris was removed by centrifugation. Protein concentration was determined with the Bio-Rad protein assay. Cell lysates were boiled for $5 \mathrm{~min}$ in $1 \mathrm{X}$ SDS sample buffer $(50 \mathrm{mM}$ Tris- $\mathrm{HCl} \mathrm{pH} 6.8,12.5 \%$ glycerol, $1 \%$ sodium dodecylsulfate, $0.01 \%$ bromophenol blue) containing $5 \% \beta$-mercaptoethanol. Adrenal cell lysate (positive control for MC2R) was obtained from VWR.

Immunofluorescence microscopy. Cells were grown on Lab-Tek II chamber slides for $24 \mathrm{~h}$. Appropriate treatments were added as described in the figure legends. Cells were then rinsed 3x in PBS and fixed in buffered $4 \%$ paraformaldehyde (30 min). Following fixation, cells were rinsed $2 \mathrm{x}$ in $\mathrm{dH}_{2} \mathrm{O}$ and transferred to absolute methanol for $10 \mathrm{~min}$ followed by equilibration in PBS ( $3 x$ for $3 \mathrm{~min}$ each). Before adding the primary antibody, cells were incubated in appropriate blocker (5\% normal goat serum, 2\% BSA, in PBS, pH 7.3). Rabbit polyclonal anti-MC2R (extracellular) and anti-AR (nuclear) were incubated (1:100) in blocking solution overnight at RT. Secondary, fluorescence-labeled goat anti-rabbit, IgG antibody was incubated at RT for $1 \mathrm{~h}$. Slides were mounted with Vectashield mounting medium and the preparations were sealed with clear nail polish. Epifluorescence and DIC images were acquired with a Nikon E600 microscope equipped with a Retiga EX CCD digital camera (Q Imaging, Burnaby, Canada). Images were processed and saved with NIS-Elements AR 3.2 software. Exposure times were identical among the treatment groups. Confocal images were captured with a Nikon TE2000E confocal microscope (Nikon, Mississauga, Canada) operated with EZ-C1 3.91 software.

Cyclic AMP. ACTH-induced cAMP was determined in three PCa cell lines. Briefly, PC3, DU-145, and LNCaP cells were plated in 6-well plates and allowed to attach for $16 \mathrm{~h}$. The following day, the cells were washed twice with warm Waymouth/BSA. Then fresh Waymouth/BSA containing $0.5 \mathrm{mM}$ isobutylmethylxanthine (IBMX) (a cAMP promoter 

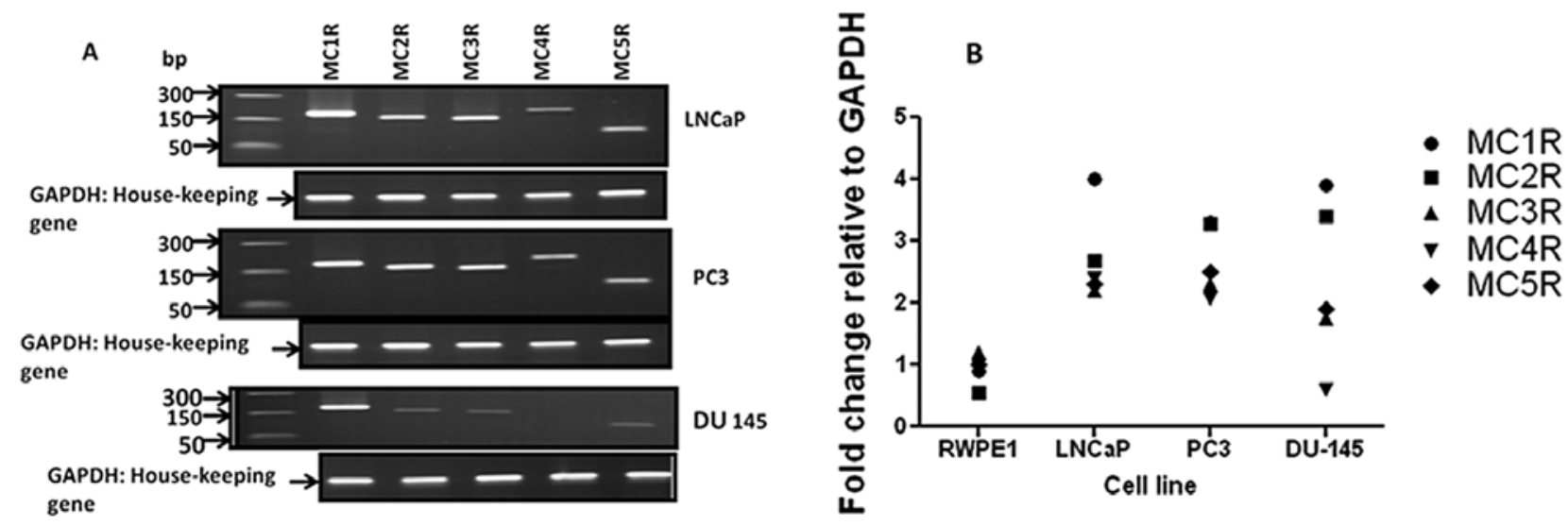

Figure 1. (A) MCR (1-5) transcripts are expressed in human PCa cell lines. Conventional reverse transcriptase polymerase chain reaction (RT-PCR) showing MCR (1-5) mRNA expression in human androgen-dependent LNCaP, and androgen-independent PC3 and DU-145 human PCa cells. Lane 1, DNA molecular weight markers; lanes 2-6, MCR (1-5), respectively. GAPDH, a house keeping gene, was included as control for RT-PCR and for assessment of gel loading (B) Quantitative real-time analysis showing comparative levels of MCRs (1-5) in RWPE1 normal human prostate cells and in LNCaP, PC3, and DU-145, PCa cell lines.

and a non-specific inhibitor of cAMP and cGMP phosphodiesterases, Sigma) was added to each well. After 15 min of incubation at $37^{\circ} \mathrm{C}$, different concentrations of $\mathrm{ACTH}$ or $\left[\mathrm{Nle}^{4}\right.$, D-Phe $\left.{ }^{7}\right]-\alpha$-melanocyte stimulating hormone (NDP-MSH) were added and the cells were incubated for another hour. Final concentrations of ligands are indicated in Fig. 7. Intracellular cAMP was extracted using $0.5 \mathrm{~N}$ perchloric acid containing $180 \mu \mathrm{g} / \mathrm{ml}$ theophylline (Sigma), and measured using radioimmunoassay (32). All determinations were performed in triplicate. The cAMP levels were calculated as fold increase over basal levels of each cell line. Maximal response (Rmax) and $\mathrm{EC}_{50}$ values were calculated with GraphPad software.

Statistical analysis. Data from cell culture MTT cell viability assay were expressed as the mean \pm SD. The data were analyzed by Student's t-test, one-way ANOVA followed by Dunnett's post hoc test (Graph Pad Prism 5.0, San Diego, CA). P<0.05 was considered to indicate statistically significant differences. Statistical analysis for the real-time PCR data was performed using a modification of the $\Delta \Delta \mathrm{Ct}$ method $(\Delta \Delta \mathrm{Ct})$ as described in our previous study (33) .

\section{Results}

MCR (1-5) transcripts are expressed in PCa cell lines. We used conventional and real-time RT-PCR analysis to determine that MCR (1-5) transcripts are expressed in LNCaP and PC 3 cells. DU-145 cells expressed the MCR 1-3, and 5 transcripts, but did not express MC4R mRNA (Fig. 1A). Comparative real-time PCR analysis of MCR (1-5) showed higher expression in cancer cells compared with RWPE-1 normal prostate epithelial cells (Fig. 1B). Similar to conventional RT-PCR data, the MC4R level in DU-145 cancer cells was undetectable by the real-time PCR technique. Table I shows the expected PCR product size and the accession numbers of MCR genes in Genbank.

ACTH (MC2R specific ligand) increases cell proliferation in human PCa cells and SHU9119 (a universal MCR blocker) partially blunts this effect. Because increased GPCRs expression in PCa cells was thought to provide cell survival signal

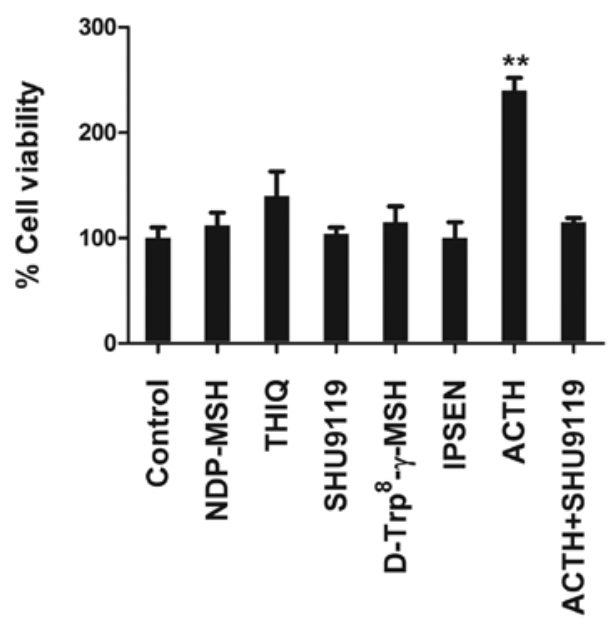

Figure 2. Among several MCR synthetic agonists and antagonists used to stimulate cell proliferation, only ACTH treatment $\left(10^{-5}\right.$ final concentration) increased cell proliferation in LNCaP cells. The ACTH effect was partially blocked by SHU9119, a universal MCR blocker. The potent selective agonists D-Trp ${ }^{8}-\gamma$-MSH (for MC3R), THIQ (for MC4R), and NDP-MSH (for all MCR except MC2R) failed to induce cell proliferation. Similarly, SHU9119, a universal MCR blocker, and IPSEN i5, MC4R inverse agonist, did not alter cell viability/proliferation. Values are representative of three experiments. Similar results for ACTH were obtained in PC3 and DU-145 (see Fig. 3).

in PCa (34). We used several MCR synthetic peptide agonists and antagonists in cell viability/proliferation assays to test the function of the MCRs in PCa cells. These compounds were: NDP-MSH, the potent $\alpha-M S H$ analogue for all MCRs except MC2R; THIQ, the highly selective agonist of MC4R; D-Trp 8 $\gamma$-MSH, the highly selective agonist of MC3R; SHU9119, a universal MCR antagonist; and Ipsen 5i, an inverse MC4R agonist. In LNCaP, only ACTH induced proliferation and enhanced cell viability (Fig. 2). Similar findings were obtained in PC3 and DU-145 cells (data not shown). The stimulatory effects of ACTH were concentration-dependent in both androgen-dependent $\mathrm{LNCaP}$ and androgen-independent PC3 and DU-145 cells (Fig. 3A and B). The maximal cell proliferation increase was between 55 and $170 \%$ above the control. Cell proliferation increased incrementally with continuous 

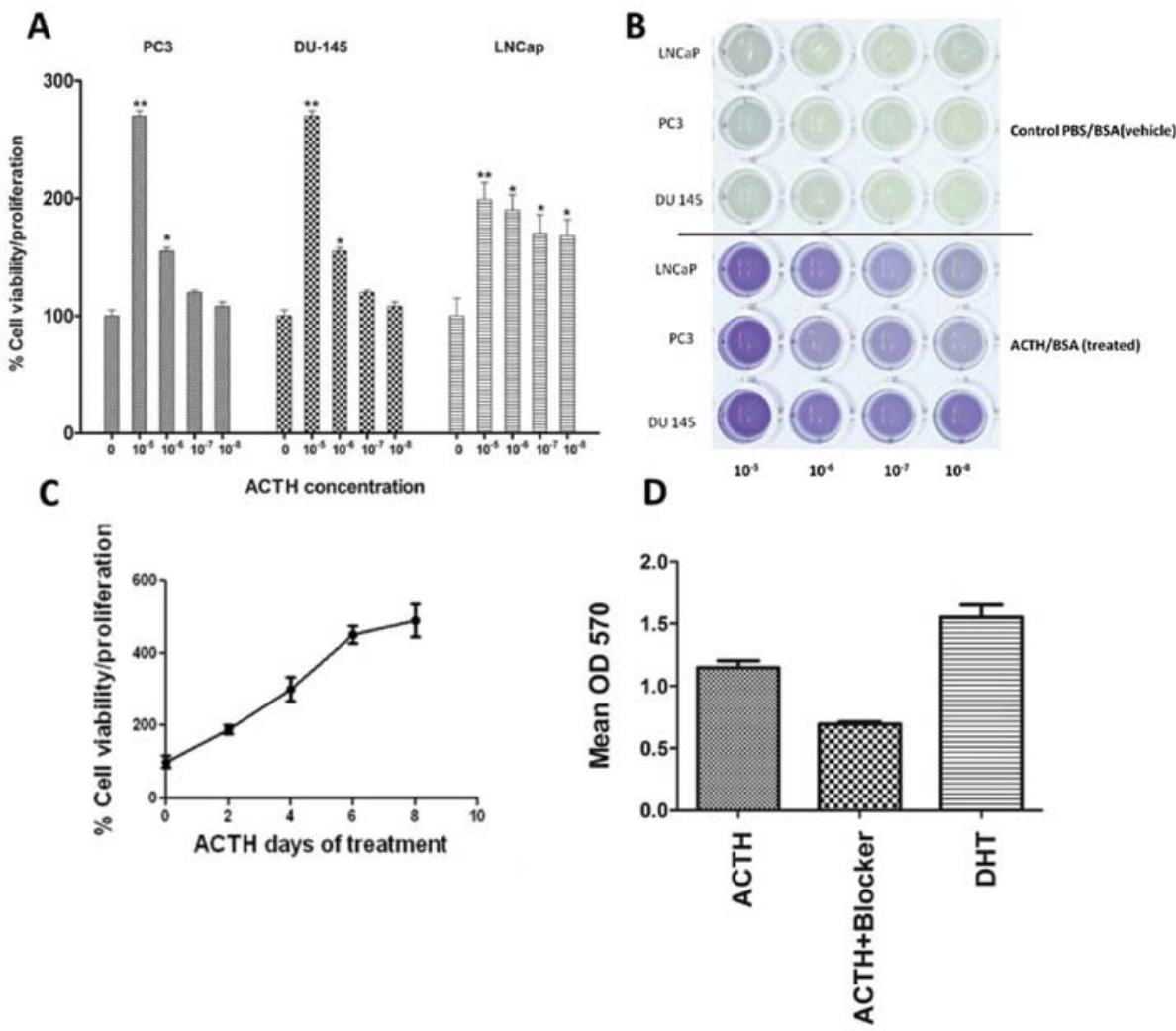

Figure 3. (A) ACTH increases cell proliferation in a dose-dependent manner in androgen-dependent LNCaP, and androgen independent PC3 and DU-145. Cell viability was determined by MTT viability/proliferation assay using four ACTH concentrations. Values are representative of three experiments. (B) Representative MTT assay plate image showing concentration-dependent increase in cell proliferation (indicated by conversion of MTT to purple color) in LNCaP, PC3, and DU-145. Note the color change from light yellow in wells treated with PBS/BSA vehicle to purple in ACTH/BSA-treated wells. (C) Kinetics of PC3 proliferative responses to ACTH stimulation overtime. Cells were treated with $10^{-5} \mathrm{ACTH}$ for $2,4,6$, or 8 days. (D) ACTH-induced cell proliferation was partially reversed by MCR SHU9119 (a universal MCR blocker) in LNCaP cells. DHT is a potent AR activator and was used as positive control for cell proliferation.

A
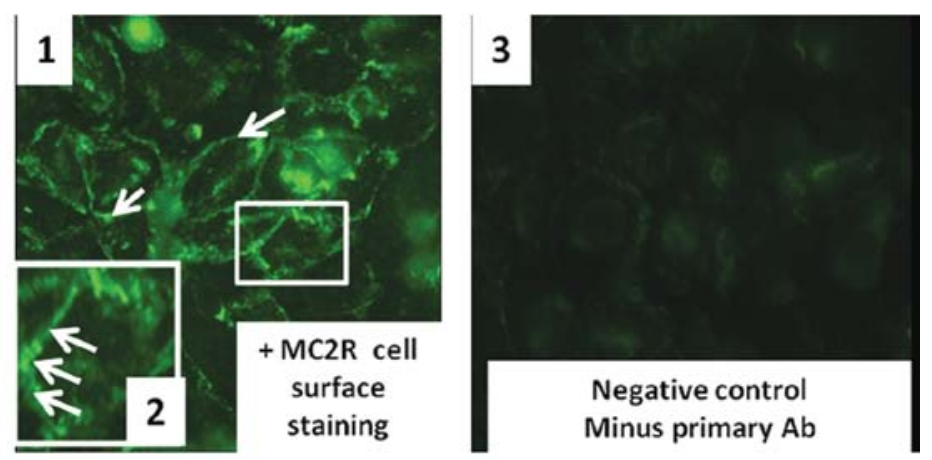

B

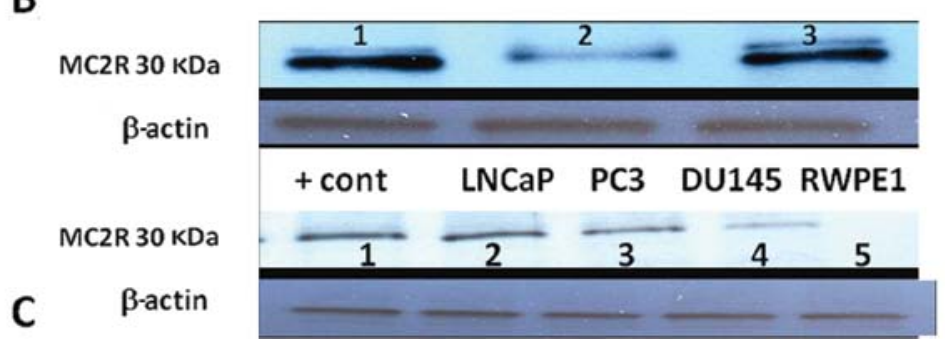

Figure 4. (A) Immunohistochemical localization of MC2R in PC3 using rabbit anti-melanocortin receptor 2 (extracellular) antibody captured with fluorescence-labeled goat anti-rabbit secondary antibody. Note specific cell surface expression of MC2R in panel (1). Insert (panel 2) is the magnification of a cell membrane to show punctuated and discrete plasma membrane sub-domains. Little or no staining is present in negative control (minus primary Ab, panel 3).

(B) MC2R protein is present in $\mathrm{LNCaP}$ lysate as shown by western blotting and was masked by MC2R specific peptide blocker. Lane 1, MC2R protein; lane 2, MC2R band was partially abolished when MC2R antibody was pre-incubated with a control MC2R peptide; lane 3, positive MC2R control (35 $\mu \mathrm{g}$ adrenal cell lysate). (C) MC2R protein in LNCaP, PC3, DU-145 PCa cell lines and RWPE1 normal prostate cells. Lane 1, adrenal lysate (positive control); lane 2, LNCaP; lane 3, PC3; lane 4, DU-145; lane 5, RWPE1. 
ACTH treatment for up to 8 days (Fig. 3C). Treatment with the universal MCR blocker SHU9119 inhibited cell proliferation in ACTH treated LNCaP cells by approximately 50\% (Fig. 3D).

Indirect immunocytochemistry showed a punctate label pattern on PC3 cell membranes (Fig. 4A). The labeling pattern was similar to that described in transfected HEK293-related cells (293/FRT cell line) (29). Immunoblots incubated with MC2R antibody that was pre-absorbed with commercially available MC2R polypeptide showed no band (Fig. 4B). Routine western blot analysis showed higher MC2R protein expression in LNCaP, PC3, DU-145 cells compared with normal non-tumorigenic RWPE2 prostate cells (Fig. 4C).

ACTH proliferative effect is additive with DHT stimulation. Owing to the fact that adrenal steroids, dehydroepiandrosterone (DHEA), dehydroepiandrosterone sulfate (DHEAS), and androstenedione, are induced by ACTH and are linked to $\mathrm{PCa}$ recurrence, especially in patients undergoing long-term androgen ablation $(6,35)$, we investigated a possible interaction with ACTH and androgen. ACTH stimulation of LNCaP cells in combination with DHT, a potent AR agonist, induced a 150-200\% increase in cell proliferation compared with ACTH or DHT alone (Fig. 5).

ACTH treatment enhances AR protein nuclear translocation and AR protein expression in $L N C a P$. Production of cAMP/ PKA via GPCR activation can transactivate AR in prostate carcinoma (25). Therefore, we considered whether ACTH stimulation of LNCaP cells affected the amount of nuclear AR protein expression. Immunocytochemistry showed increased AR nuclear signal intensity following ACTH treatment compared to that seen in vehicle treated cells (Fig. 6A). Similarly, western blot analysis of ACTH treated LNCaP cells confirmed an increase in total AR expression (Fig. 6B and C). Forskolin, a cAMP inducer and AR activator that mimics ACTH action, and DHT, a potent AR agonist, were included as positive controls. The results of these experiments indicate that ACTH treatment enhances AR nuclear translocation and increases AR protein in PCa cells (Fig. 6A-C).

ACTH treatment increases cAMP concentration. Cyclic AMP is a critical mediator of cell proliferation and differentiation; it induces cAMP-dependent PKA production in various cell types. As shown in Fig. 7, all three PCa cell lines responded to ACTH stimulation with concentration-dependent cAMP production increases. The mean $\mathrm{EC}_{50}$, from three independent experiments was, $1.79 \mu \mathrm{M}$ for PC-3; $3.20 \mu \mathrm{M}$ for DU-145; and $3.67 \mu \mathrm{M}$ for $\mathrm{LNCaP}$. The highest ACTH concentration $(10 \mu \mathrm{M})$ increased the cAMP level 81-fold in PC3 cells, 111-fold in DU-145 cells, and 52-fold in LNCaP cells. There was no increase in cAMP production following NDP-MSH treatment ( $\mathrm{n}=3$, data not shown).

MRAP- $\alpha$ and $-\beta$ are expressed in PCa cells. As coexpression of MC2R accessory protein isoforms $\alpha$ and $\beta$ (MRAP- $\alpha$ and $-\beta$ ) is required for cAMP signaling via $M C 2 R$ in some cells such as human embryonic kidney 293 cells (36), we examined whether MRAP- $\alpha$ and MRAP- $\beta$ mRNA transcripts are expressed in LNCaP, PC3, and DU-145 PCa cells. RT-PCR analysis showed amplification of both transcripts in

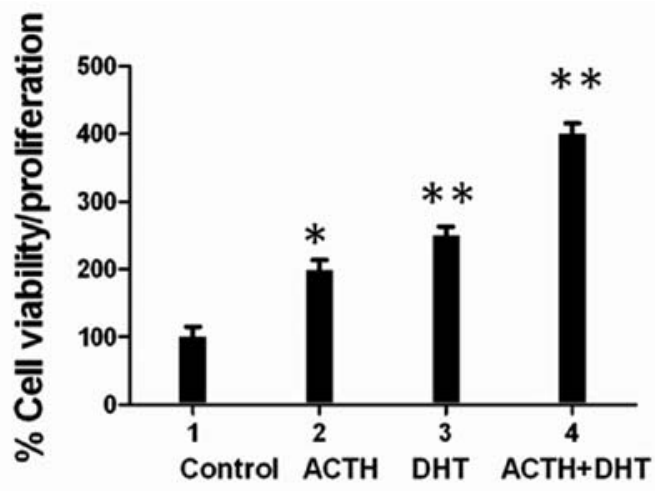

Figure 5. ACTH (MC2R specific ligand) stimulatory effect on cell proliferation was additive to DHT in LNCaP human PCa cells which express functional AR. ACTH $=10^{-5}$, DHT $=1 \mathrm{nM}$. Data represent values obtained from three experiments. ${ }^{*} \mathrm{P}<0.05 ;{ }^{* *} \mathrm{P}<0.001$.

all three cell lines (Fig. 8). Positive control cDNA from the adrenal gland was amplified in parallel for validation of the PCR products.

\section{Discussion}

The findings reported here strongly link the ACTH-MC2R signaling pathway to $\mathrm{PCa}$ progression. This conclusion is based on several observations. Transcripts for MCRs, as well as MC2R protein, are expressed in LNCaP, PC 3 and DU-145 human PCa cell lines. ACTH, the primary ligand for MC2R, increased cell proliferation/viability in the androgen dependent LNCaP and the androgen-independent PC3 and DU-145 cells by upregulating cAMP. ACTH-MC2R-induced cell proliferation was partly abrogated by SHU9119, a universal MCR blocker. Finally, AR was transactivated and its nuclear translocation increased in $\mathrm{LNCaP}$ cells following treatment with exogenous ACTH.

Physiological and sub-nanomolar ACTH concentrations stimulated PCa cell proliferation under serum-free conditions and in the absence of an exogenous cAMP inducer. The mitogenic effect was above $100 \%$ that of the control. NDP-MSH, a potent $\alpha \mathrm{MSH}$ analogue and agonist of all but MC2R, as well as D-Trp ${ }^{8}-\gamma$-MSH and THIQ, highly selective agonists for MC3R- and MC4R respectively, failed to stimulate cell proliferation. These results were predictable since it is known that all five MCRs respond to ACTH (37) but, among the MCRs, MC2R binds only ACTH and lacks affinity for other melanocortins. The other MCRs are, however, activated by both MSH and ACTH. The additional observation that the universal MCR blocker SHU9119 reduces but does not cancel the mitogenic effect of ACTH suggests that proliferation is at least partially mediated by MC2R.

Such trophic effects of ACTH were reported in human adrenal gland cells and skin melanocytes (38). Several studies documented what was referred to as 'ectopic ACTH syndrome' whereby several cancers from endocrine sites such as the pituitary gland (39) and diverse non-endocrine sites including the prostate gland, lung, liver, pancreas, and neuronal tissue, secrete ACTH that drives cell proliferation in these tissues (40-42).

ACTH-driven cell proliferation was likely mediated via cAMP induction since ACTH is known to bind MC2R, 
A
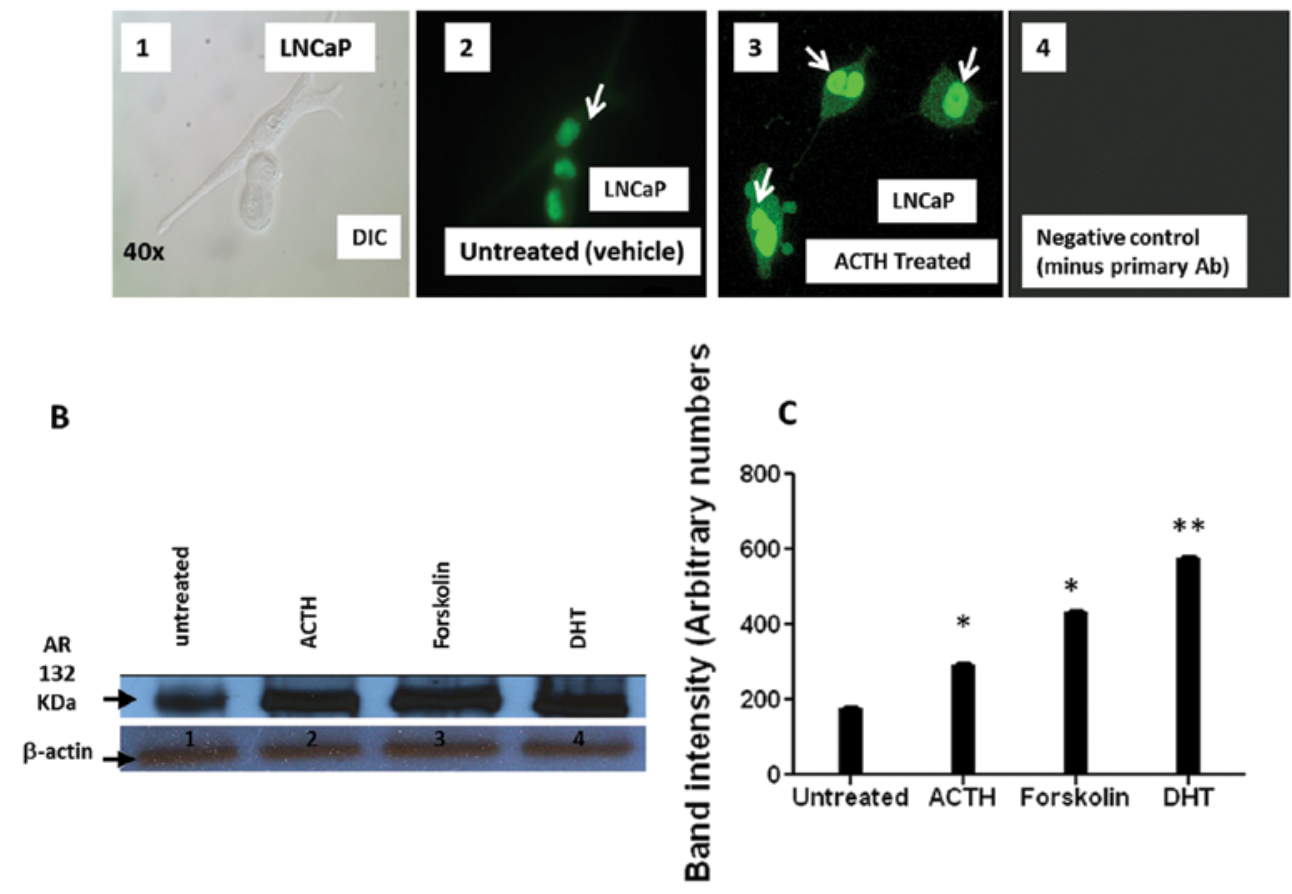

Figure 6. (A) ACTH treatment $\left(10^{-5}\right)$ increased the nuclear staining intensity of the AR protein in LNCaP that have functional AR. Panel 1, DIC, differential interference contrast for cells in panel 2. Panel 2, AR expression in LNCaP cells treated with PBS/BSA vehicle. Panel 3, AR nuclear staining in cells treated with ACTH. Note the increased fluorescence intensity of AR nuclear staining in panel 3 (arrows). Panel 4, negative control (minus MC2R primary antibody). (B) ACTH treatment upregulated AR protein expression in LNCaP. Western blot analysis of AR protein expression in untreated LNCaP cells (lane 1), in cells treated with ACTH (lane 2), cAMP inducer Forskolin (lane 3), or DHT (lane 4). $\beta$-actin was included as control to monitor gel loading. (C) Quantitative analysis of band intensity in (B) using Unscan software. Data for (C) were obtained from two experiments.

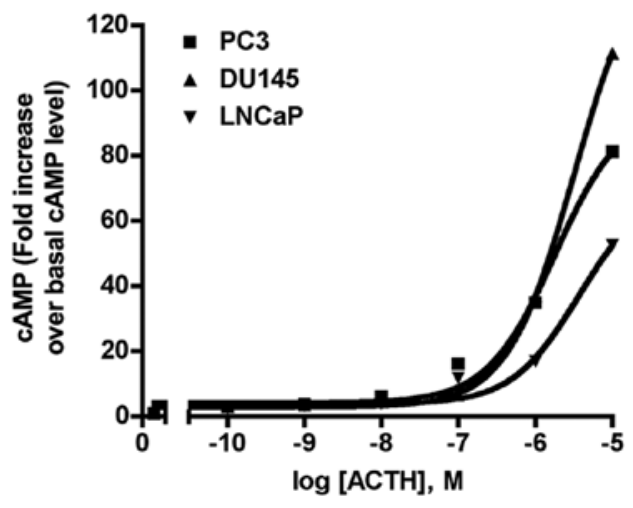

Figure 7. Treatment of LNCaP, PC3, and DU-145 cells with ACTH increased cAMP production in a concentration-dependent manner. Intracellular cAMP levels were measured using radioimmunoassay. Results are expressed as fold increase over basal cAMP level of each cell line.

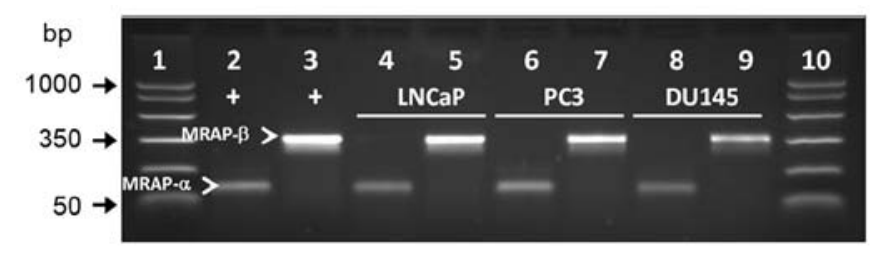

Figure 8. MRAP- $\alpha$ and MRAP- $\beta$ (arrowheads) are expressed in LNCaP, PC3, and DU-145 human PCa cell lines. PCR product sizes for MRAP- $\alpha$ and MRAP- $\beta$ are 100 and $350 \mathrm{bp}$, respectively. Lane 1, molecular weight DNA markers; lanes 2 and 3, positive controls for MRAP- $\alpha$ and MRAP- $\beta$, respectively (adrenal DNA). Lanes 4 and 5, LNCaP; lanes 6 and 7, PC3; lanes 8 and 9, DU-145. Lane 10, molecular weight DNA markers. a $\mathrm{G}$ protein-coupled receptor, which subsequently activates adenylyl cyclase and results in cAMP production (43). The significant cAMP increases at high ACTH concentrations (above $1 \mu \mathrm{M}$ ) reported here indicated that an endogenous ACTH receptor is present in all three PCa cell lines. Since NDP-MSH is a superpotent agonist for other MCRs, except for MC2R, we reasoned that if the PCa cells cannot respond to NDP-MSH, the observed ACTH-dependent cAMP production in our three PCa cell lines should be caused by direct MC2R activation by ACTH. Indeed, there was no cAMP production by NDP-MSH stimulation (data not shown), suggesting that MC2R is expressed in all three cell lines.

The enhanced transactivation of AR via this pathway suggests cross-talk between MC2R and AR and supports the view of a critical downstream AR role in driving PCa cell growth in androgen-dependent and androgen-independent $\mathrm{PCa}$ cell growth (5). The increased AR protein expression following ACTH treatment suggests that the ACTH-MC2R signaling transduction pathway contributes to enhanced AR-DNA binding. That increased binding could promote PCa cell growth, an effect that would be particularly important under low androgen conditions. Such a conclusion is consonant with studies showing that several PCa cell GPCRs are indirectly involved in AR transactivation (13). In PCa, several GPCRs that signal through $\mathrm{G} \alpha$ s, such as prostaglandin E2 receptors (EP2 and EP4), and $\alpha$-adrenergic receptor, can transactivate AR and induce cAMP and PKA activation which, in turn, can enhance AR sensitivity to low androgen levels. Our data indicate that MC2R is another GPCR that contributes to PCa cell proliferation and survival. 
The central feature of the study reported here relates to PCa cell proliferation by ACTH-MC2R interaction that was partially abrogated by the universal MCR blocker SHU9119. This strongly suggests a paracrine role for ACTH and its $\mathrm{MC} 2 \mathrm{R}$ in regulating PCa cell growth. The fact that there are two approximately equivalent androgen sources acting in the prostate gland, namely $\mathrm{T}$ from the testis and androgen derived from the adrenal gland, makes a strong case for including MC2R blockade as part of a combination therapy that involves inhibitors of androgen and other hormones.

\section{Acknowledgements}

This study was partially supported by funds from the interdepartmental Research Grants Program, Scott-Richey Research Center and the Boshell Diabetes and Metabolic Diseases Program, College of Veterinary Medicine, Auburn University, Auburn, AL, USA.

\section{References}

1. Woolf SH: Screening for prostate cancer with prostate-specific antigen. An examination of the evidence. N Engl J Med 333: 1401-1405, 1995.

2. Oh WK, Kantoff PW: Management of hormone refractory prostate cancer: Current standards and future prospects. J Urol 160: 1220-1229, 1998.

3. Li TH, Zhao H, Peng Y, Beliakoff J, Brooks JD and Sun Z: A promoting role of androgen receptor in androgen-sensitive and -insensitive prostate cancer cells. Nucleic Acids Res 35: 2767-2776, 2007.

4. Huggins C: Endocrine-induced regression of cancers. Cancer Res 27: 1925-1930, 1967.

5. Debes JD, Tindall DJ: Mechanisms of androgen-refractory prostate cancer. N Engl J Med 351: 1488-1490, 2004.

6. Feldman BJ and Feldman D: The development of androgenindependent prostate cancer. Nat Rev Cancer 1: 34-45, 2001.

7. Nguyen MM and Wang Z: Manipulation of androgens and alterations in the androgen receptor axis in prostate cancer. Minerva Urol Nefrol 60: 15-29, 2008.

8. Grossmann ME, Huang H, Tindall DJ: Androgen receptor signaling in androgen-refractory prostate cancer. J Natl Cancer Inst 93: 1687-1697, 2001.

9. Craft N, Shostak Y, Carey M and Sawyers CL: A mechanism for hormone-independent prostate cancer through modulation of androgen receptor signaling by the HER-2/neu tyrosine kinase. Nat Med 5: 280-285, 1999.

10. Linja MJ and Visakorpi T: Alterations of androgen receptor in prostate cancer. J Steroid Biochem Mol Biol 92: 255-264, 2004.

11. Vis AN and Schröder FH: Key targets of hormonal treatment of prostate cancer. Part 1: the androgen receptor and steroidogenic pathways. BJU Int 104: 438-448, 2009.

12. Culig Z, Hobisch A, Cronauer MV, et al: Androgen receptor activation in prostatic tumor cell lines by insulin-like growth factor-I, keratinocyte growth factor and epidermal growth factor. Eur Urol 27 (Suppl 2): 45-47, 1995.

13. Dorsam RT and Gutkind JS: G-protein-coupled receptors and cancer. Nat Rev Cancer 7: 79-94, 2007.

14. Daaka Y: G proteins in cancer: The prostate cancer paradigm. Sci STKE: re2, 2004.

15. Mendiratta P, Armstrong AJ and George DJ: Current standard and investigational approaches to the management of hormonerefractory prostate cancer. Rev Urol 9: S9-S19, 2007.

16. Raj GV, Barki-Harrington L, Kue PF and Daaka Y: Guanosine phosphate binding protein coupled receptors in prostate cancer: A review. J Urol 167: 1458-1463, 2002.

17. Xia C, Ma W, Wang F, Hua SB, Liu M: Identification of a prostate-specific G-protein coupled receptor in prostate cancer. Oncogene 13: 5903-5907, 2001.

18. Wang J, Weng J, Cai Y, Penland R, Liu M, Ittmann M: The prostate-specific G-protein coupled receptors PSGR and PSGR2 are prostate cancer biomarkers that are complementary to alphamethylacyl-CoA racemase. Prostate 1: 847-857, 2006.
19. Weng J, Wang J, Cai Y, et al: Increased expression of prostatespecific G-protein-coupled receptor in human prostate intraepithelial neoplasia and prostate cancers. Int J Cancer 113: 811-818, 2005.

20. Yowell CW and Daaka Y: G protein-coupled receptors provide survival signals in prostate cancer. Clin Prostate Cancer 1: 177-181, 2002.

21. Sun L, Luo J, Mackey LV, et al: Investigation of cancer cell lines for peptide receptor-targeted drug development. J Drug Target 19: 719-730, 2011.

22. Gutkind JS: Regulation of mitogen-activated protein kinase signaling networks by $\mathrm{G}$ protein-coupled receptors. Sci STKE: re1, 2000 .

23. McDonnell TJ, Troncoso P, Brisbay SM, et al: Expression of the Protooncogene bcl-2 in the Prostate and Its Association with Emergence of Androgen-independent Prostate Cancer. Cancer Res 52: 6940-6944, 1992.

24. Kasbohm EA, Guo R, Yowell CW, et al: Androgen receptor activation by $\mathrm{G}(\mathrm{s})$ signaling in prostate cancer cells. J Biol Chem 280: 11583-11589, 2005.

25. Merkle D and Hoffmann R: Roles of cAMP and cAMP-dependent protein kinase in the progression of prostate cancer: Cross-talk with the androgen receptor. Cell Signal 23: 507-515, 2011.

26. Luscombe CJ, French ME, Liu S, et al: Prostate cancer risk: Associations with ultraviolet radiation, tyrosinase and melanocortin-1 receptor genotypes. Br J Cancer 85: 1504-1509, 2001.

27. Wisse BE, Frayo RS, Schwartz MW and Cummings DE: Reversal of cancer anorexia by blockade of central melanocortin receptors in rats. Endocrinology 142: 3292-3301, 2001.

28. Chen W, Kelly MA, Opitz-Araya X, Thomas RE, Low MJ and Cone RD: Exocrine gland dysfunction in MC5-R-deficient mice: Evidence for coordinated regulation of exocrine gland function by melanocortin peptides. Cell 91: 789-798, 1997.

29. Roy S, Pinard S, Chouinard L and Gallo-Payet N: Adrenocorticotropin hormone (ACTH) effects on MAPK phosphorylation in human fasciculata cells and in embryonic kidney 293 cells expressing human melanocortin 2 receptor (MC2R) and MC2R accessory protein (MRAP) $\beta$. Mol Cell Endocrinol 336: 31-40, 2011.

30. Alwani RA, Neggers SJ, van der Klift M, et al: Cushing's syndrome due to ectopic ACTH production by (neuroendocrine) prostate carcinoma. Pituitary 12: 280-283, 2009.

31. Mansour M, Schwartz D, Judd R, et al: Thiazolidinediones/ PPAR $\gamma$ agonists and fatty acid synthase inhibitors as an experimental combination therapy for prostate cancer. Int J Oncol 38: 537-546, 2011.

32. Tao YX, Huang H, Wang ZQ, Yang F, Williams JN and Nikiforovich GV: Constitutive activity of neural melanocortin receptors. Methods Enzymol 484: 267-279, 2010.

33. Mansour M, White D, Wernette C, et al: Pancreatic neuronal melanocortin-4 receptor modulates serum insulin levels independent of leptin receptor. Endocrine 37: 220-230, 2010.

34. Marinissen MJ and Gutkind JS: G-protein-coupled receptors and signaling networks: emerging paradigms. Trends Pharmacol Sci 22: 368-376, 2001.

35. Labrie F, Belanger A, Dupont A, Luu-The V, Simard J and Labrie C: Science behind total androgen blockade: from gene to combination therapy. Clin Invest Med 16: 475-492, 1993.

36. Roy S, Rached M and Gallo-Payet N: Differential regulation of the human adrenocorticotropin receptor [melanocortin-2 receptor $(\mathrm{MC} 2 \mathrm{R})]$ by human MC2R accessory protein isoforms alpha and beta in isogenic human embryonic kidney 293 cells. Mol Endocrinol 21: 1656-1669, 2007.

37. Cone RD: Studies on the physiological functions of the melanocortin system. Endocr Rev 27: 736-749, 2006.

38. Abdel-Malek Z, Swope VB, Suzuki I, et al: Mitogenic and melanogenic stimulation of normal human melanocytes by melanotropic peptides. Proc Natl Acad Sci USA 92: 1789-1793, 1995.

39. Melmed S: Pathogenesis of pituitary tumors. Nat Rev Endocrinol 7: 257-266, 2011.

40. Liddle GW, Island DP, Ney RL, Nicholson WE and Shimizu N: Nonpituitary neoplasms and cushing's syndrome: Ectopic 'adrenocorticotropin' produced by nonpituitary neoplasms as a cause of Cushing's syndrome. Arch Intern Med 111: 471-475, 1963.

41. Gewirtz G and Yalow RS: Ectopic ACTH production in carcinoma of the lung. J Clin Invest 53: 1022-1032, 1974.

42. Singer W, Kovacs K, Ryan N and Horvath E: Ectopic ACTH syndrome: clinicopathological correlations. J Clin Pathol 31: 591-598, 1978.

43. Halkerston ID: Cyclic AMP and adrenocortical function. Adv Cyclic Nucleotide Res 6: 99-136, 1975. 